\title{
Usos de la cánula nasal de alto flujo para pacientes con covid-19. ¿Cómo funciona, cuáles son sus indicaciones? ¿Es segura en los pacientes con insuficiencia respiratoria aguda hipoxémica?*
}

\author{
Gustavo Adolfo Hincapié Díaz ${ }^{a}$ Claudia Liliana Echeverría González ${ }^{b}$ Luisa \\ Fernanda Enciso Bahamón ${ }^{c}$
}

Resumen: la pandemia por coronavirus tipo 2 o SARS-COV-2 tiene en jaque al sistema sanitario mundial dada su alta tasa de contagio; ha hecho colapsar todos los sistemas de salud y ha puesto en evidencia la escasez de recursos como los elementos de protección y la cantidad insuficiente de ventiladores en relación con el número de pacientes con falla respiratoria aguda hipoxémica. Por estas razones, se ha tenido que recurrir a diferentes opciones, una de ellas la cánula de alto flujo, que permite administrar oxígeno a alto flujo a los pacientes con hipoxemia severa. Durante la pandemia por Covid-19, se ha generado la inquietud acerca de que este dispositivo, como todos los dispositivos que permiten la administración de oxígeno, produce gran dispersión de aerosoles y la posibilidad de contagio del personal de la salud, lo cual ha llevado a desestimar su uso y utilizar de manera casi exclusiva la cánula nasal convencional y la ventilación mecánica en sujetos infectados. De manera que en este trabajo, se realizó una revisión de la literatura que recoge los mecanismos de acción, su seguridad para el personal de salud y las indicaciones del uso de la cánula nasal de alto flujo en los pacientes infectados por COVID-19 así como sus ventajas; entre ellas, ser dispositivo ahorrador de ventiladores mecánicos, lo que puede ser muy útil en el manejo de los pacientes en caso de un pico epidémico en Colombia.

Palabras clave: COVID-19; pandemia; insuficiencia respiratoria hipoxémica; cánula nasal de alto flujo Recibido: 12/05/2020. Aceptado: 30/06/2020.

* Artículo de investigación.

a MD, internista y neumólogo, coordinador del servicio de Neumología del Hospital Militar Central. Profesor titular de la Universidad Militar Nueva Granada, Bogotá (Colombia). Correo electrónico: gustavo.hincapie@unimilitar.edu.co ORCID: https://orcid.org/0000-0002-5375-4776

b MD, pediatra. Magíster en Epidemiología. Directora de posgrado de la Universidad Militar Nueva Granada, Bogotá (Colombia). Correo electrónico: clecheverriag@gmail.com

c MD, internista. Residente de Neumología de la Universidad Militar Nueva Granada, Bogotá (Colombia). Correo electrónico: luisitaenciso@gmail.com 
Disponible en línea: 04/06/2021

Cómo citar: Hincapié Díaz GA, Echeverría González CL, Enciso Bahamón LF. Usos de la cánula nasal de alto flujo para pacientes con covid-19. ¿Cómo funciona, cuáles son sus indicaciones? ¿Es segura en los pacientes con insuficiencia respiratoria aguda hipoxémica?. Rev. Med. [Internet]. 4 de junio de 2021 [citado 4 de junio de 2021];28(2):25-4. Disponible en: https://revistas.unimilitar.edu.co/index. $\mathrm{php/rmed/article/view/5101}$

\title{
Uses of the high-flow nasal cannula for covid-19 patients. How does it work an what are its indications? Is it safe or patients with acute hypoxic respiratory failure?
}

\begin{abstract}
SARS-CoV-2 pandemic has held a threat over the world health system due to its high transmission rate. It has made all health systems collapse and has made evident the lack of resources such as personal protection elements and the insufficient number of ventilators for the number of patients with acute hypoxic respiratory failure. Due to his reasons, many options have been used as an alternative, being the high-flow nasal cannula one of them since it allows administering high-flow oxygen to patients with severe hypoxemia. During the CoviD-19 epidemic, a concern has raised regarding this device as well as all devices used to supply oxygen to patients since they produce great dispersion of aerosols and the possibility of transmission to clinicians and staff. This has led to reject its use and give almost exclusive preference to the use of conventional cannula and mechanical ventilators with infected patients. Therefore, this study reviews the literature dealing with the high-flow nasal cannula action mechanisms, its safety for health workers and its indications for patients infected with coviD-19, as well as its advantages; amongst them, being a device that prevents the use of mechanical ventilators, which can be useful when managing patients in case of an epidemiological peak in Colombia.
\end{abstract}

Keywords: COVID-19; pandemic; hypoxic respiratory failure; high-flow nasal cannula

\section{Usos da cânula nasal de alto fluxo para pacientes com covid-19. Como funciona e quais suas indicações? É segura nos pacientes com insuficiência respiratória aguda hipoxêmica?}

Resumo: a pandemia pelo coronavírus tipo 2, SARS-CoV-2, põe em xeque o sistema sanitário mundial tendo em vista sua alta taxa de contágio. Além disso, tem feito colapsar todos os sistemas de saúde e evidenciado a escassez de recursos como os elementos de proteção e a quantidade insuficiente de ventiladores para o número de pacientes com insuficiência respiratória aguda hipoxêmica. Por essas razões, recorre-se a diferentes opções, uma delas a cânula de alto fluxo, que permite administrar oxigênio a alto fluxo aos pacientes com hipoxemia severa. Durante a pandemia pela covid-19, muitas dúvidas são geradas sobre esse dispositivo, como todos os dispositivos que permitem o manejo do oxigênio, produz grande dispersão de aerossóis e a possibilidade de contágio da equipe de saúde, o que tem levado a desestimar seu uso e a utilizar de maneira quase exclusiva a cânula nasal convencional e a ventilação mecânica em sujeitos infectados. Assim, neste trabalho, foi realizada uma revisão da literatura que coleta os mecanismos de ação, sua segurança para a equipe de saúde e as indicações do uso da cânula nasal de alto fluxo nos pacientes com covid-19, bem como suas vantagens; entre elas, ser dispositivo que dispensa ventiladores mecânicos, o que pode ser muito útil no manejo dos pacientes caso haja um pico epidêmico na Colômbia.

Palavras-chave: covid-19; pandemia; insuficiência respiratória hipoxêmica; cânula nasal de alto fluxo 


\section{Introducción}

A finales del año 2019 en la ciudad de Wuhan, China, se reporta epidemia de un nuevo coronavirus que se denominó Coronavirus del Síndrome Respiratorio Grave 2 (sARS- COV-2) (1). Posteriormente se da el nombre de enfermedad por coronavirus 2019 (CovID-19). El 11 de marzo de 2020, la Organización Mundial de la Salud (oMs), dada la presencia del virus en más de 120000 casos en 114 países y más de 4000 muertes declara la pandemia mundial (2).

De acuerdo con los informes de China, la prevalencia de la insuficiencia respiratoria aguda hipoxémica (IRAH) en pacientes con COVID-19 es del $19 \%$. De estos, del $4 \%$ al $13 \%$ recibieron ventilación mecánica no invasiva (VMNI) y alrededor del $12 \%$ requieren ventilación mecánica invasiva (vMI). No está aún establecida la verdadera incidencia de IRAH en pacientes con COVID-19, pero se ha estimado que el $14 \%$ de los enfermos desarrollan enfermedad grave y $5 \%$ requieren tratamiento en unidad de cuidado intensivo (UCI) con VMI. En los estudios descriptivos de China se reporta que de 52 pacientes críticos con Covid-19, el $67 \%$ desarrollaron síndrome de dificultad respiratoria aguda (SDRA), y 33 de ellos (63,5 \%) recibieron tratamiento con oxígeno por cánula nasal de alto flujo (CNAF), como soporte de la hipoxemia (3). La terapia con oxígeno de alto flujo a través de una cánula nasal puede ofrecer una alternativa en pacientes con hipoxemia (4).

La neumonía grave causada por el SARS-COV-2 constituye una amenaza a los recursos sanitarios en todo el mundo, principalmente en pacientes que desarrollan IRAH. Desde los brotes que se presentaron de SARS (severe acute respiratory syndrome) en el 2003, ha existido un interés que viene cada vez en aumento sobre la capacidad de los agentes infecciosos de transmitirse por aerosoles, con la limitante que la visualización de los flujos de aires es difícil para el ojo humano. Para ello se ha utilizado trazadores de partículas nebulizadas sobre superficies sólidas o contenidas en humos con posterior fotografía para tratar de calcular la distancia alcanzada por las partículas (5). Por lo tanto, la utilización de diferentes dispositivos para dispensar oxígeno ha generado la inquietud de un posible riesgo de infección al personal de la salud debido a la aerosolización de partículas. Por esta razón se ha contraindicado en las instituciones del país la terapia con micronebulizaciones y en algunos centros la ventilación mecánica no invasiva y la máscara Venturi.

La administración de oxígeno constituye la primera línea terapéutica en pacientes con insuficiencia respiratoria aguda, la cual se ofrece a través de máscaras faciales o cánula nasal. Hay varios inconvenientes asociados a estas interfases, debido a que la cantidad de oxígeno suministrada no es suficiente para corregir la hipoxemia. La entrega de oxígeno suele ser limitada dado que el máximo flujo que se puede lograr es de $15 \mathrm{~L} / \mathrm{min}$ en caso de la máscara y el oxígeno suministrado debe succionar aire del medioambiente, efecto Venturi, para que esta dilución de gases ingrese a la vía aérea (6).

Desde hace varios años se ha venido trabajando en la forma de aumentar la humidificación del aire dispensado por los dispositivos fuentes de aire, ya que esta constituía el paso limitante para administrar oxígeno a alto flujo dispensado a través de la nariz. La adición de aire lo suficientemente tibio y húmedo al gas respirado ha permitido que se dispense oxígeno de alto flujo a los pacientes. Primero se utilizó en los recién nacidos pretérminos en escenarios donde se hubiese tenido que ventilar estos pacientes con CPAP (continuous positive air pressure, por sus siglas en inglés) y luego se trasladó a los pacientes adultos en diferentes escenarios como edema pulmonar, enfermedad pulmonar obstructiva crónica y posextubación, entre otras. A continuación, se procederá a explicar su mecanismo de acción en cinco pasos. Primero, lava el espacio muerto de la nasofaringe que contribuye a mejorar la fracción alveolar de los gases tanto del dióxido de carbono como del oxígeno. Segundo, la distensibilidad de la nasofaringe es significativa en el esfuerzo inspiratorio versus el esfuerzo espiratorio, por lo que la CNAF ayuda a mejorar esta relación para que sea más pareja, y así atenuar la resistencia asociada a la nasofaringe y eliminar el trabajo relacionado con la respiración. Tercero, la administración de aire húmedo y caliente hacia las vías conductoras mejora la conductancia y la distensibilidad pulmonar comparado con el aire frío 
y seco. Cuarto, el aire húmedo y caliente que pasa por la nasofaringe reduce la tasa metabólica asociada al acondicionamiento gaseoso que se da en condiciones usuales y por último titula el alto flujo a través de la nasofaringe para dar presión positiva de distensión para el reclutamiento pulmonar (7).

La terapia con oxígeno de alto flujo a través de una cánula nasal puede ofrecer una alternativa en pacientes con hipoxemia. Se trata de una terapia que emplea la humidificación activa para permitir la entrega de flujo hasta de $60 \mathrm{~L} / \mathrm{min}$ con concentraciones de oxígeno que van desde el $21 \%$ hasta el $100 \%$ además de calentar el aire a $37^{\circ} \mathrm{C}$ con humedad relativa del $100 \%$, y se entrega a través de un circuito inspiratorio calentado para evitar pérdida de calor y condensación y al paciente a través de la interfase que son cánulas nasales de silicona, más largas que las convencionales (Figura 1).

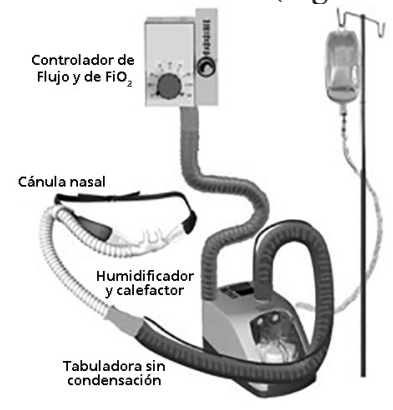

Figura 1. Cánula nasal de alto flujo

Fuente: elaboración propia.

Es un sistema que consta de un analizador y controlador de flujo y FiO2, que envía oxígeno a un sistema de calefacción y humidificación. Posteriormente se envía la mezcla de oxígeno y aire por tubuladura corrugada que impide la condensación y mantiene la calefacción. Al paciente se le entrega el oxígeno por cánulas nasales.

Los dientes de la cánula deben ajustarse a las fosas nasales para evitar que entre aire del medioambiente, problema que se presenta en las cánulas nasales convencionales. La exhalación es al aire libre. La CNAF adicionalmente reduce el espacio muerto fisiológico, proporciona bajos niveles de PEEP y disminuye la taquipnea por reducción del trabajo respiratorio; aunque los niveles de PEEP entregados son variables y discutidos dependiendo de varios factores del paciente como la obesidad y la talla, o si es un paciente adulto o pediátrico. Además, parece disminuir la precarga por aumento de la presión negativa intratorácica y produce paralelamente reclutamiento de alvéolos (8).

Para ofrecer un adecuado tratamiento del paciente con IRAH en el contexto de COVID-19, es importante detectar en forma oportuna el compromiso pulmonar, la gravedad de la hipoxemia, el compromiso sistémico asociado y la presencia de choque. La oxigenación con CNAF puede por lo tanto ser utilizada como una alternativa terapéutica en pacientes con IRAH quienes no tienen indicación absoluta de vmi. Sin embargo, todas las formas de oxigenación suplementaria pueden potencialmente aerosolizar patógenos respiratorios. Por esta razón la escogencia del soporte respiratorio para pacientes con COVID-19 debe tener una adecuada valoración de la relación de riesgo/beneficio de diseminación nosocomial del virus, pero en fase de mitigación realizar intubación orotraqueal en forma temprana implica la utilización de una cama de UCI y de un ventilador mecánico, que pueden desabastecerse durante el pico de la pandemia. Por esto, la terapia alternativa que se escoja debe cumplir con el requisito de ser efectiva para mejorar la hipoxemia, el trabajo respiratorio y ser segura para el personal de salud (9).

A la fecha no existen estudios en los cuales se evalúe como desenlace la efectividad, no inferioridad o superioridad de la cánula de alto flujo versus otras formas de dispensación de oxígeno en los pacientes covid y existe controversia sobre su uso. Las diferentes sociedades científicas tienen opiniones opuestas acerca de su uso. La AARC (American Association for Respiratory Care) y la campaña de supervivencia de sepsis para el tratamiento de los pacientes críticamente enfermos de COVID-19 recomiendan en pacientes con hipoxemia temprana el uso con precaución de CNAF, incluso sobre la oxigenoterapia convencional (recomendación débil) y preferirla sobre la ventilación mecánica no invasiva (recomendación débil). El paciente en tratamiento con VMNI O CNAF, se debe tener en estricta monitorización de deterioro respiratorio y 
ofrecer intubación temprana (declaración como la mejor práctica clínica) (10).

El Servicio Nacional de Salud del Reino Unido (National Health Service, NHS) (11) en un documento guía del uso de soporte no invasivo en pacientes con COVID-19 desaconsejan la utilización de la CNAF por falta de eficacia y riesgo de diseminación de partículas infecciosas; sin embargo, no hacen referencia a las fuentes de dicha información y no amplían los argumentos para divulgar esta recomendación. No hace parte de estrategias de ventilación, dado que es solo una forma más de suministrar oxígeno con alto flujo. En todas las publicaciones aclara que es para minimizar los riesgos de contaminación nosocomial y se aconseja tener cámara de presión negativa con al menos 10 ciclos/hora de intercambio de aire y elementos de protección personal.

En la Tabla 1 se hace un resumen de las recomendaciones.

Tabla 1. Resumen de recomendaciones de los diferentes grupos de asociaciones mundiales sobre el uso de CNAF Y VMNI

\begin{tabular}{l|ll} 
Asociación & NIV & CNAF \\
\hline OMS & Sí & Sí \\
\hline SSC & Sí & Sí \\
\hline NHS & Sí & No \\
\hline ANZICS & No & Sí
\end{tabular}

Abreviaciones. OMs: Organización Mundial de la Salud. ANIZCS: Australian and New Zealand Intensive Care Society. Ssсм: Surviving Sepsis Campaign.

Fuente: elaboración propia.

La OMs en su documento dice que la CNAF no crea dispersión del aire exhalado y por lo tanto el riesgo de dispersión de partículas es bajo, sin embargo, recomienda que el personal de salud debe permanecer a 2 metros de distancia, de acuerdo con el manejo clínico de la infección respiratoria aguda grave cuando se sospecha una nueva infección por coronavirus, la cual es una orientación provisional (12).

Como se mencionó previamente, los casos severos de neumonía e insuficiencia respiratoria aguda por SARS-COV-2 u otros coronavirus que han producido brotes de epidemias como SARS, MERS (Middle East Respiratory Syndrome) o incluso influenza aviar A(H7N9) y A(H5N1) pueden llevar a falla respiratoria con altas tasas de mortalidad, ante lo cual se requiere dispensación de oxígeno sea por cánula nasal convencional, máscaras faciales, ventilación mecánica no invasiva (VMNI) o ventilación mecánica invasiva (VMI). Todas las formas de dispensación generan aerosoles o gotas, lo cual lleva a brotes de infecciones nosocomiales. Durante el brote de SARS-COV se identificaron como factores de riesgo para la diseminación rápida del virus en las instituciones de salud: la resucitación cardiopulmonar, la distancia entre camas de pacientes menor a un metro, personal de salud que trabaje a pesar de estar sintomático, vMNI y la oxigenoterapia mayor a 6 litros/minuto. Factores de riesgo que se identificaron igualmente durante el brote de MERS. Estos pacientes se deben tratar preferiblemente en cámaras de presión negativa para controlar la infección y su posible diseminación, y entender que la distancia de dispersión y la dirección del aire exhalado cuando se utiliza la CNAF y CPAP a diferentes tasas de flujo de aire pueden, de forma potencial, ocasionar grandes cantidades de aerosoles. Por lo que se ha estudiado dicha dispersión y dirección del aire exhalado con el empleo de CNAF en cámaras aisladas con presión negativa con tasas de 16 recambios/hora en simuladores de pacientes de alta fidelidad que contienen modelos pulmonares y de vía aérea reales, con variación de las tasas de oxígeno de alto flujo entre $10 \mathrm{a} 60 \mathrm{~L} / \mathrm{min}$ y la aplicación de CPAP entre 5 a $20 \mathrm{~cm} \mathrm{H} 20$, con la que se simulan pulmones normales, con daño leve y daño pulmonar severo marcando con trazadores el aire exhalado para demostrar que la capacidad de dispersión cambia según el daño pulmonar: cuando el pulmón es sano el aire exhalado es capaz de viajar una distancia de $65 \mathrm{~mm}$ cuando se administra oxígeno a $10 \mathrm{~L} / \mathrm{min}$ y aumenta hasta $172 \mathrm{~mm}$ con oxígeno a $60 \mathrm{~L} / \mathrm{min}$, mientras que el pulmón con daño moderado disminuye su velocidad de exhalación a $72 \mathrm{~mm}$ cuando se le administra oxígeno a $60 \mathrm{~L} / \mathrm{min}$ y en casos de daño pulmonar severo es capaz de aerosolizar a una distancia de $48 \mathrm{~mm}$. Sin embargo, es importante tener en cuenta la dispersión lateral y en este mismo estudio se encontró 
que esta dispersión es casi que obvia si la cánula se ajusta de forma adecuada al tubo que dispensa el oxígeno, pero si la interfaz se desconecta la dispersión se evidencia hasta $620 \mathrm{~mm}$ lateralmente con CNAF a $60 \mathrm{~L} / \mathrm{min}(4)$.

A pesar de la preocupación inicial por lo médicos en todo el mundo por la dispersión de aerosoles, los sistemas de CNAF no fueron utilizados inicialmente, aunque dada la alta eficacia para mantener la oxigenación y de acuerdo con la evidencia disponible con baja contaminación, en estudios experimentales realizados con CNAF al colocar tapabocas quirúrgico al paciente, disminuye el riesgo de dispersión de aerosol al toser o estornudar a menos de $20 \mathrm{~cm}$, lo que además es un beneficio adicional, ya que no es posible utilizar un tapabocas sobre una máscara de Venturi (13). Incluso existen ya sociedades como la Sociedad Española de Neumología y Cirugía Torácica que recomiendan esta práctica ante la duda de la existencia real de dispersión (14).

Estudios en los que se tomaron modelos de experimentación y mediante el uso de luz láser se podía visualizar las partículas de humos exhaladas; se pudo evaluar a diferentes valores de $\mathrm{FiO} 2$ y flujo de oxígeno la distancia de dispersión en centímetros (13).

Se revisa la evidencia y se hace un resumen de la distancia de dispersión, como se observa en la Tabla 2.

Tabla 2. Dispersión vs. dispositivo de oxígeno

\begin{tabular}{lll} 
Dispositivo de oxígeno & & $\begin{array}{l}\text { Distancia de } \\
\text { dispersión cm }\end{array}$ \\
\hline HFNC & $60 \mathrm{~L} \mathrm{~min}^{-1}$ & $17.2 \pm 3.3$ \\
\hline & $30 \mathrm{~L} \mathrm{~min}^{-1}$ & $13.0 \pm 1.1$ \\
\hline & $10 \mathrm{~L} \mathrm{~min}^{-1}$ & $6.5 \pm 1.5$ \\
\hline Máscara simple & $15 \mathrm{~L} \mathrm{~min}^{-1}$ & $11.2 \pm 0.7$ \\
\hline & $10 \mathrm{~L} \mathrm{~min}^{-1}$ & $9.5 \pm 0.6$ \\
\hline Máscara de no reinhalación & $10 \mathrm{~L} \mathrm{~min}^{-1}$ & $24.6 \pm 2.2$ \\
\hline Máscara Venturi en Fi02 0.4 & $6 \mathrm{~L} \mathrm{~min}^{-1}$ & $39.7 \pm 1.6$ \\
\hline Máscara Venturi en Fi02 0.35 & $6 \mathrm{~L} \mathrm{~min}^{-1}$ & $27.2 \pm 1.1$ \\
\hline
\end{tabular}

HFNC: cánula nasal de alto flujo. $\mathrm{FiO}_{2}$ : fracción inspirada de oxígeno. Fuente: elaboración propia.
En la literatura consultada se encontraron resultados diferentes en cuanto a los desenlaces de mortalidad y requerimiento de intubación orotraqueal. Algunos estudios demuestran la disminución de la tasa de intubación orotraqueal (15). El flujo alto genera bajos niveles de presión positiva en las vías aéreas superiores, $\mathrm{FiO} 2$ variable, susceptible de ajustar a necesidad, cuya propiedad de lavado de $\mathrm{CO} 2$ disminuye el espacio muerto fisiológico con reducción del trabajo respiratorio. En pacientes con IRAH independiente de la causa, se ha demostrado que el oxígeno de alto flujo permite mayor comodidad y oxigenación comparado con la máscara facial (16).

Debido a la disminución de la necesidad de intubación orotraqueal en insuficiencia respiratoria, la mortalidad es menor en los pacientes tratados con CNAF, al evitar los efectos adversos de la VMI. El oxígeno con CNAF mostró reducción en la gravedad de la disnea y una disminución de la frecuencia respiratoria (17).

Rochwerg et al. (18) realizan una revisión sistemática y un metaanálisis con los cuales se evaluó la seguridad y eficacia de la CNAF en pacientes con IRAH. No se encontró efecto sobre mortalidad (RR 0,94 CI 0,67 - 1,31), cuando se comparó con la oxigenoterapia convencional en 1647 sujetos se logró demostrar su capacidad de reducir la necesidad de ventilación mecánica (RR 0,85 CI 0,74 - 0,99). La reducción absoluta del riesgo (RAR) fue de $4,4 \%$ con NNT de 23. Además, la terapia con CNAF puede reducir la necesidad de escalar la terapia (RR 0,71 CI 0,51 - 0,98). La RAR fue de 9,5\% y el NNT, de $11 \%$. No tuvo efecto sobre la estancia en UCI. El efecto de evitar la intubación y vMI fue consistente con estudios anteriores. El hecho de no realizar vMI disminuye el riesgo de infecciones nosocomiales, delirio y enfermedad tromboembólica. Estos desenlaces se asocian a menos costos relacionados con el tratamiento.

Ni et al. (19) en una revisión sistemática y un metaanálisis, que incluyó ensayos clínicos controlados y aleatorizados, comparan la CNAF y la oxigenoterapia convencional (oc) o VMNI en 1084 pacientes, 506 de ellos tratados con CNAF, 208 con VMNI y 370 con oxigenoterapia convencional. La CNAF reduce la necesidad de intubación 
orotraqueal con una tasa de intubación orotraqueal de $50 \%$ en el grupo de vMNI vs. $20 \%$ en el grupo de CNAF; además de obtener un mayor número de días libres de uso de ventiladores de 28 días. Se encontró disminución de la mortalidad: un $22,2 \%$ en pacientes con vMNI vs. un $5 \%$ en pacientes con CNAF. Estos hallazgos se explican probablemente por una adecuada ventilación/minuto y oxigenación suficiente además de su capacidad de entregar una concentración de oxígeno que es constante, mientras que la oc no puede entregar el $\mathrm{FiO} 2$ necesario a un paciente con taquipnea dado que se diluye en el ambiente el oxígeno inspirado. Por ende, se produce una disminución tanto en la frecuencia como en el trabajo respiratorio y mejora la sincronía toracoabdominal, lo que hace que la respiración que es más lenta y profunda puede aliviar la disnea del paciente por la vía neural, que la activación de los mecanismos reflejos y neuronales que igualmente disminuyen la frecuencia y el trabajo respiratorios. Además, que el sistema de humificación preserva la función de la mucosa y facilita el aclaramiento de secreciones, lo que reduce el riesgo de atelectasia y mejora la oxigenación.

Existe la necesidad de predecir el paciente que va a fallar a este tipo de terapia con CNAF, por lo que se desarrolló el índice Rox definido como la relación entre la saturación de oxígeno y la fracción inspiratoria de oxígeno o $\mathrm{SaO} 2 / \mathrm{FiO} 2$ sobre la frecuencia respiratoria (FR). En el trabajo original se eligieron pacientes con neumonía de cualquier etiología que fueran incapaces de mantener saturaciones por encima del $92 \%$ (a nivel del mar) o con FR por encima de 25 respiraciones por minuto, que no tuvieran indicación inmediata de intubación o que tuvieran indicación de no reanimación, en 5 unidades de cuidado intensivo en España y Francia. Gracias a curvas de Roc (regression operating curve) se encontró que por encima de un valor de 4,88, independientemente del tiempo de uso inicial: a las 2, 6 y 12 horas de terapia con CNAF los pacientes tienen mayor probabilidad de éxito y los que tienen un valor inferior se asocian con mayor riesgo de intubación en los modelos de regresión de Cox. Los pacientes que no logran subir dicho índice en el mismo tiempo de evaluación, igualmente se asocian con fracaso a la terapia (20). Ya existe incluso un trabajo en pacientes con neumonía por SARS-COV-2 confirmado en el que se reevaluaron las curvas de Kaplan-Meyer en un estudio único retrospectivo en pacientes mayores de 18 años, en el cual se les midió el índice Rox varias veces al día, y se encontró que el valor promedio de este índice para predecir falla a la terapia con CNAF fue de 5,37, valor superior a la descripción inicial antes de la pandemia, como se comentó antes. Los pacientes que se encuentren por encima de este valor tienen menor riesgo de fracaso a la terapia con el alto flujo (21).

A pesar de la preocupación inicial de los médicos en todo el mundo por la dispersión de aerosoles, los sistemas de CNAF no fueron utilizados inicialmente; no obstante, dada la alta eficacia para mantener la oxigenación y de acuerdo con la evidencia disponible con baja contaminación, en estudios experimentales realizados con CNAF, al colocar tapabocas quirúrgico al paciente, disminuye el riesgo de dispersión de aerosol al toser o estornudar a menos de $20 \mathrm{~cm}$, lo que además es un beneficio adicional, ya que no es posible utilizar un tapabocas sobre una máscara de Venturi.

\section{Conclusiones}

Todos los sistemas de oxígeno son posibles aspersores de partículas, mas no generadores, esto significa que la producción de gotas o aerosoles en el paciente y el flujo e interfaz son los que incrementan la distancia de goteo o dispersión del aerosol. Por lo tanto, todos los sistemas constituyen un riesgo para el personal de salud que está en contacto con el paciente si la distancia es inferior a un metro.

La inclusión de CNAF se propone como una alternativa para manejar a pacientes que no responden a la oxigenoterapia convencional, pero en quienes no tienen criterios de intubación se aclara que no es una estrategia de ventilación sino de aporte de oxígeno a alto flujo. Como opinión de los autores, se considera que este es el gran problema de los estudios ya que incluyen la CNAF en la ventilación mecánica no invasiva cuando no lo es.

En el marco del riesgo de contaminación, cabe destacar que no es inocuo, aunque se encuentra en el mismo espectro de riesgo de los otros sistemas, 
pero con muy buenos resultados en falla hipoxémica, lo que permite disminuir la tasa de intubación.

Esta alternativa, que es de fácil manejo por el personal de salud y que tiene aprobación en COVID-19 por la OMS y la Organización
Panamericana de la Salud (PAHO, por sus siglas en inglés), en fase de mitigación de pandemia COVID-19 permitirá liberar ventiladores mecánicos que deben estar disponibles para pacientes más críticos.

\section{Referencias}

1. Wei-jie G, Zheng-yi N, Yu H, Wen-hua L, Chun-quan O, Jian-xing H et al. Clinical characteristics of coronavirus disease 2019 in China. N Engl J Med. 2020;382(18):1708-1720. Dor: https://doi.org/10.1056/NEJMoa2002032

2. Organización Mundial de la Salud. Alocución de apertura del director general de la OMS en la rueda de prensa sobre la COVID-19 celebrada el 11 de marzo de 2020. [Internet]. 2020. Disponible en: https://bit.ly/31OQsP1

3. Xiaobo Y, Yuan Y, Jiqian X, Huaqing S, Jia'an X, Hong L et al. Clinical course and outcomes of critically ill patients with SARS-Cov-2 pneumonia in Wuhan, China: a single-centered, retrospective, observational study. The Lancet. 2020;8(5):475-481. Dor: https://doi.org/10.1016/S2213-2600(20)30079-5

4. Hui DS, Chow BK, Lo T, Tsang OTY, Ko FW, Ng SS et al. Exhaled air dispersion during high-flow nasal cannula therapy versus CPAP via different masks. Eur Respir J. 2019;53(4):1802339. DoI: https://doi.org/10.1183/13993003.023392018

5. Ip M, Tang JW, Hui DSC, Wong ALN, Chan MTV, Joynt GM et al. Airflow and droplet spreading around oxygen masks: a simulation model for infection control research. Am J Infect Control. 2007;35(10):684-689. DoI: https://doi. org/10.1016/j.ajic.2007.05.007

6. Scheaua FD. Theoretical approaches regarding the Venturi effect. Hidraulica. 2016;(3):69-72. Disponible en: https:// bit.ly/3e0OpfE

7. Dysart K, Miller TL, Wolfson MR, Shaffer TH. Research in high flow therapy: mechanisms of action. Respir Med. 2009;103(10):1400-1405. DoI: https://doi.org/10.1016/j.rmed.2009.04.007

8. Lodeserto FJ, Lettich TM, Rezaie SR. High-flow nasal cannula: mechanisms of action and adult and pediatric indications. Cureus. 2018;10(11):e3639. DoI: https://doi.org/10.7759/cureus.3639

9. Whittle J, Pavlov I, Sacchetti AD, Atwood C, Rosenberg MS. Respiratory support for adult patients with COVID-19. JACEP Open. 2020;1(2):95-101. DoI: https://doi.org/10.1002/emp2.12071

10. Alhazzani W, Møller MH, Arabi YM, Loeb M, Gong MN, Fan E et al. Surviving sepsis campaign: guidelines on the management of critically ill adults with coronavirus disease (COVID-19). Intensive Care Med. 2020;(46):854-887. DOI: https://doi.org/ggqp2p

11. National Health Service (NHS). Guidance for the role and use of non-invasive respiratory support in adult patients with COVID-19 (confirmed or suspected). Version 3. 2020. Disponible en: https://bit.ly/3jzRFjo

12. World Health Organization. Operational considerations for case management of COVID-19 in health facility and community. Interim guidance World Health Organization. Pediatr Med Rodz. 2020;16(1):27-32. Dor: https://doi. org/10.15557/PiMR.2020.0004

13. Li J, Fink JB, Ehrmann S. High-flow nasal cannula for COVID-19 patients: low risk of bio-aerosol dispersion. Eur Respir J. 2020;55(5):2000892. DOI: https://doi.org/10.1183/13993003.00892-2020

14. Cinesi C, Peñuelas Ó, Luján M, Egea C, Masa JF, García J et al. Recomendaciones de consenso respecto al soporte respiratorio no invasivo en el paciente adulto con insuficiencia respiratoria aguda secundaria a infección por SARSCov-2. Arch. bronconeumol. 2020;56(2):11-18. DoI: https://doi.org/10.1016/j.arbres.2020.03.005

15. Parke RL, McGuinness SP, Eccleston ML. A preliminary randomized controlled trial to assess effectiveness of nasal high-flow oxygen in intensive care patients. Respir Care. 2011;56(3):265-270. DoI: https://doi.org/10.4187/respcare.00801 
16. Sztrymf B, Messika J, Mayot T, Lenglet H, Dreyfuss D, Ricard JD. Impact of high-flow nasal cannula oxygen therapy on intensive care unit patients with acute respiratory failure: a prospective observational study. J Crit Care. 2012;27(3):324.e9-324.e13. DoI: https://doi.org/10.1016/j.jcrc.2011.07.075

17. Frat JP, Thille AW, Mercat A, Girault C, Ragot S, Perbet S et al. High-flow oxygen through nasal cannula in acute hypoxemic respiratory failure. N Engl J Med. 2015;(372): 2185-2196. DoI: https://doi.org/10.1056/NEJMoa1503326

18. Rochwerg B, Granton D, Wang DX, Helviz Y, Einav S, Frat JP et al. High flow nasal cannula compared with conventional oxygen therapy for acute hypoxemic respiratory failure: a systematic review and meta-analysis. Intensive Care Med. 2019;(45):563-572. DoI: https://doi.org/10.1007/s00134-019-05590-5

19. Ni YN, Luo J, Yu H, Liu D, Liang BM, Liang ZA. The effect of high-flow nasal cannula in reducing the mortality and the rate of endotracheal intubation when used before mechanical ventilation compared with conventional oxygen therapy and noninvasive positive pressure ventilation. A systematic review and meta-analysis. Am J Emerg Med. 2018;36(2): 226-233. DoI: https://doi.org/10.1016/j.ajem.2017.07.083

20. Roca O, Caralt B, Messika J, Samper M, Sztrymf B, Hernández G et al. An index combining respiratory rate and oxygenation to predict outcome of nasal high-flow therapy. Am J Respir Crit Care Med. 2019;199(11):1368-1376. DoI: https://doi.org/10.1164/rccm.201803-05890C

21. Zucman N, Mullaert J, Roux D, Roca O, Ricard JD. Prediction of outcome of nasal high flow use during COVID-19-related acute hypoxemic respiratory failure. Intensive Care Med. 2020;(46):1924-1926. DoI: https://doi.org/10.1007/ s00134-020-06177-1 
